\title{
Method of the Year: spatially resolved transcriptomics
}

\author{
Nature Methods has crowned spatially resolved transcriptomics Method of the Year 2020.
}

\section{Vivien Marx}

$\mathrm{f}$ a researcher is making a smoothie, it might be snack time. Or it could be the moment to prepare a sample for bulk RNA sequencing, in which tissue is homogenized and analyzed to yield averaged gene expression from the mRNAs in a tissue's cells - its transcriptome. When single-cell RNA sequencing (scRNA-seq) was developed, it brought a more fine-grained assessment of each cell's transcriptome. In scRNA-seq, researchers dissociate cells from tissue to, for example, discern cell types on the basis of gene expression. Working with single cells is more like digging into a fruit salad than a smoothie, says Hongkui Zeng, who directs the Allen Institute for Brain Science. Now, with spatially resolved transcriptomic methods, scientists can get transcriptomic data and know the positional context of those cells in a tissue ${ }^{1-3}$. "Fruit tart is spatial transcriptomics," says Bosiljka Tasic, an Allen Institute researcher who was interviewed jointly with Zeng. "You know exactly where each piece of fruit is and what is the relationship of each piece of fruit to the other," she says. Given how much one can learn from single cells and spatially resolved cells, the 'smoothie' approach is fast becoming passé, she says. It's not yet routine for spatial analysis to deliver transcriptome-wide information of all single cells, but the field is fast moving in that single-cell direction. Biology, says Sten Linnarsson, a Karolinska Institute biologist, "is all about context." Spatial single-cell transcriptomics is the next wave after single-cell analysis and, he says, will be particularly useful to labs studying human disease, which often starts with single cells and spreads spatially. "And if I can dream, in a few years we'll have spatiotemporal single-cell 'omics in living tissues," which brings single-cell developments full circle.

\section{Welcome, family}

Labs keen on quantifying mRNAs in their spatial context have embraced the family of methods that deliver spatially resolved transcriptomic data in neuroscience, cancer research or developmental biology, among other fields. "I think the future is bright,"

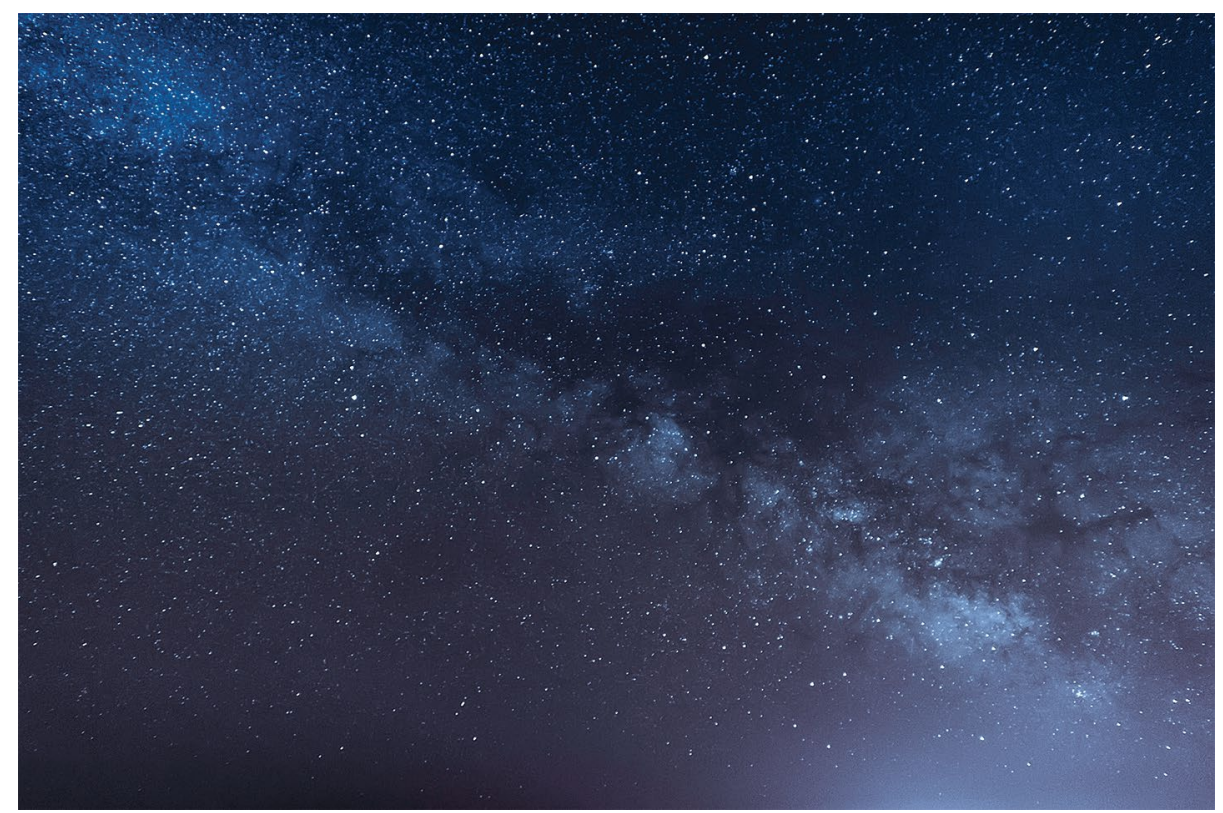

Starry skies invite space exploration. In transcriptomics, spatial resolution opens up new worlds too. Credit: bjdlzx/Getty Images

says Jay Shendure from the University of Washington. He is most excited about ways to apply these methods to obtain spatially resolved atlases of development that combine cell states, histories and fates. "It's a very active field," says Zeng, with techniques that open up new kinds of experimental opportunities. Harvard University's Xiaowei Zhuang, a Howard Hughes Medical Institute investigator, has always lived in the world of imaging. She and her lab developed a super-resolution microscopy method, stochastic optical reconstruction microscopy (STORM). When single-cell genomics emerged, she began thinking, dreaming at night, and working on a way to bring together imaging and genomics to "get the best sides of both worlds." Methods, especially those that began to emerge from around 2012 onward for capturing spatial information alongside single-cell sequence, have been melding the previously separate worlds of imaging, sequencing and molecular characterization, says Alexander van Oudenaarden, a physicist turned biologist who directs the Hubrecht Institute in Utrecht. He and his lab have combined single-cell and spatial transcriptomics to compare gene expression in gastruloids and mouse embryos. The research focus on single cells has nineteenth-century roots, such as the observation by Rudolf Virchow that disease begins in cells, not whole organisms. A decade ago, as van Oudenaarden and others peered into microscopes to study cells as they develop, grow and migrate in growing organisms, "we were always a little jealous of the sequencing world." He and his team could make time-lapse movies and capture complex processes, but only label gene expression with a handful of fluorescent proteins. Genomics labs could measure thousands of genes. To do this with tissues, he says, they "put them in a blender." Now it's become possible to assess many single cells and many genes and keep track of the cells' tissue context, their spatial attributes. 


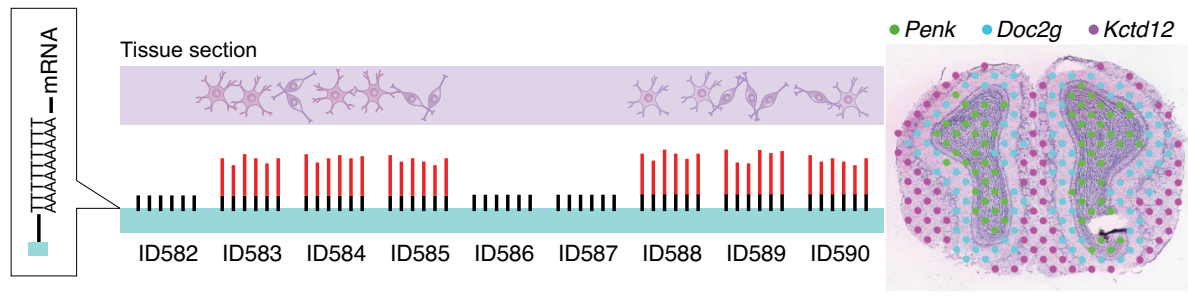

Researchers in Sweden developed an approach in which fixed, stained tissue is imaged, permeabilized and the mRNAs attach to an array of barcoded oligos. The RNAs are reverse-transcribed; the cDNAs are sequenced and yield spatially resolved transcriptomic information. Credit: Adapted with permission from ref. ${ }^{4}$, AAAS
"There are all kinds of smart ways to keep the spatial information," he says. "It's a very exciting technology."

As Joakim Lundeberg from KTH Royal Institute of Technology's Science for Life Laboratory and colleagues note $^{3}$, spatial techniques can be divided into those that involve gene expression analysis on microdissected tissues and those that involve in situ hybridization, in situ sequencing, in situ capturing, and computational reconstruction of spatial data. Single-molecule fluorescence in situ hybridization (smFISH) is "the beginning of the hybridization-based approaches" with spatial techniques, says Zeng. In this method, multiple oligonucleotides carry fluorescent labels and bind to an RNA molecule. smFISH yields a quantitative mRNA readout with "a near $100 \%$ detection sensitivity," note researchers from the Allen Institute, Science for Life Laboratory and Karolinska Institute ${ }^{2}$.

Patrik Ståhl from KTH explains that it was around 2009 when Karolinska Institute researcher Jonas Frisén set out to see how his lab could reap more information from the typical tissue slide, prepared with stains that date back to the nineteenth century. Frisén reached out to KTH colleague Lundeberg, where Ståhl was a PhD student. Fredrik Salmén was a master's student at KTH; Ståhl later joined the Frisén lab as a postdoctoral fellow and Salmén became a PhD student in Lundeberg's lab. A collaborative project in which Ståhl and Salmén worked closely together resulted in a spatial analysis approach whereby fixed, stained tissue is imaged and then permeabilized. The released mRNAs move and attach to an array beneath the tissue with barcoded oligonucleotides, which fixes them in the position they had in the tissue. After reverse transcription the tissue is enzymatically removed. What remains are spatially barcoded complementary DNA molecules attached to the oligo array.
Next comes sequencing of these cDNAs. The positional barcodes provide spatially resolved transcriptomic information. The scientists worked hard on an array that captures single cells. "We really wanted to go down to that level," says Salmén, who was interviewed jointly with Ståhl and is now a postdoctoral fellow in van Oudenaarden's lab. The method's resolution ended up being $100 \mu \mathrm{m}$, which is tens of cells. Among their many technical challenges was that the mRNAs could diffuse in many directions, which risked inaccurate spatial data or mixed expression patterns, says Salmén. They developed ways to avoid that. "It was a long struggle," says Ståhl but they and the team managed, and Ståhl and Salmén share first authorship on the paper ${ }^{4}$. The paper and the process of getting there - the melding of concepts and technology from microarrays, imaging, sequencing, and bioinformatics analysis based on barcodes - has shaped their science and their careers, says Ståhl.

Salmén's key idea, says Ståhl, was setting up an initial reaction with fluorescent nucleotides to render visible where the cells' mRNAs meet the array's surface probes.
That, says Ståhl, gives you something to image right where the synthesis of cDNA from the captured mRNA takes place. "You get a very nice fluorescent footprint of where everything went," he says. "That was, for us, a gigantic stepping stone into getting the rest to work," he says. This stepping stone convinced many the method had potential - even the "non-believers," says Salmén, laughing. Methods development means plenty of failure, he says; in this case, four to five years of failure. The method was commercialized through a spinout company, Spatial Transcriptomics, which was acquired by 10x Genomics in 2018 and led to the product Visium.

Imaging is inherently spatial and natively three-dimensional (3D), says California Institute of Technology researcher Long Cai, whose career has also been shaped by spatial methods. To get 3D information with a microscope "you just do $z$ sections," he says. He recalls when his Caltech colleague Barbara Wold mentioned to him how informative RNA sequencing could be. That was a decade ago, when second-generation sequencers were nascent. "If you think about it, sequencing is actually single-molecule imaging in a box," he says. A sequencer, "a single-molecule microscope," can generate 200-base-pair reads, which arise from 200 rounds of chemical reactions and imaging. Genomics methods yield cellular information, but to read out single-cell genomic information with imaging takes more labels than smFISH. Cai applied super-resolution microscopy to cram discernible fluorescent dots into an imaged cell. The dots can be tagged RNA, DNA or protein. His lab's methods seqFISH ${ }^{5}$ and seqFISH $+{ }^{6}$ use sequential rounds of hybridization to barcode mRNAs with a set

\section{Other spatial travels}

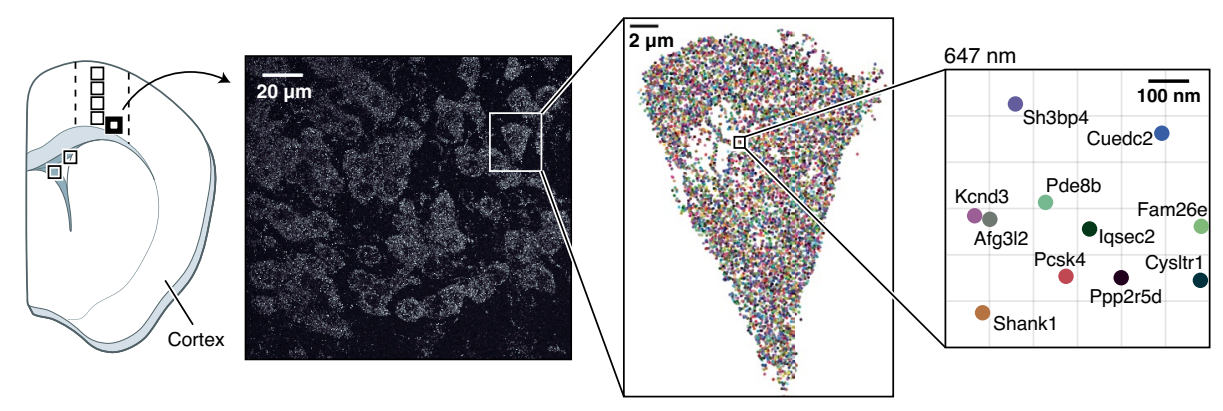

Spatial techniques help with atlas-building by localizing expressed genes. Here, seqFISH+ was used to measure 10,000 genes in mouse cortex. Credit: Cai lab, Caltech, I. Strazhnik; adapted with permission from ref. ${ }^{6}$, Springer Nature. 
of FISH probes that carry a fluorophore. The transcripts are fixed in a tissue's cells. With each round of hybridization, probes are removed for the next hybridization round with a different fluorophore. The sequence of fluorescence signals delivers spatially resolved transcriptomic data. Instead of converting imaging information into sequencing readouts, "you can do it directly, in situ," he says. He has founded a start-up called Spatial Genomics to make seqFISH commercially available.

In his view, researchers will want single-cell resolution, which scRNA-seq provides but tissue imaging, as of yet, does not. Resolution matters because in a region of interest tissues can have a mix of cell types but "a completely wrong picture" can emerge without adequate transcriptomic resolution. And if a method has low efficiency, some signals can be missed, such as the mRNAs for transcription factors expressed at ten copies or less. Cai sees as a weak point of many current spatial methods that they lead to something that "in the end you have to put in a sequencer." High-throughput genomics has had a head start, but "for almost everything sequencing can do now, there will be a spatial assay that will do the same kind of thing or better."

Evan Macosko, a physician-scientist at the Broad Institute, has been keen on using genomics to make new kinds of measurements in cells and tissues. He co-developed Drop-seq while a postdoctoral fellow in the lab of Steve McCarroll at Harvard Medical School. With Drop-seq, it takes a day to prepare thousands of sequencing libraries from many individual cells. In a microfluidic device cells travel through narrow channels and end up encapsulated in a droplet with beads, each outfitted with a unique 12-base-pair oligonucleotide barcode. Cells are lysed in the droplet and mRNAs are bar-coded and reverse-transcribed into cDNAs that are then sequenced. Together with Fei Chen, then a Broad Fellow, who co-invented expansion microscopy and who now has a lab at the Broad, Macosko developed Slide-seq ${ }^{7}$. The method draws on "DNA barcoding strategy and some of the other tricks," says Macosko. As smFISH evolved, it grew into a workhorse in his lab. The multiplexed in situ hybridization strategies are useful, but they take time and are technically demanding. "It's not something that a traditional molecular biology or cell biology lab can immediately get set up," he says. He sought a different way to get cellular or near-cellular resolution on a genome-wide scale that leverages the widespread infrastructure of Illumina-based sequencing in genomics labs. He and the team applied

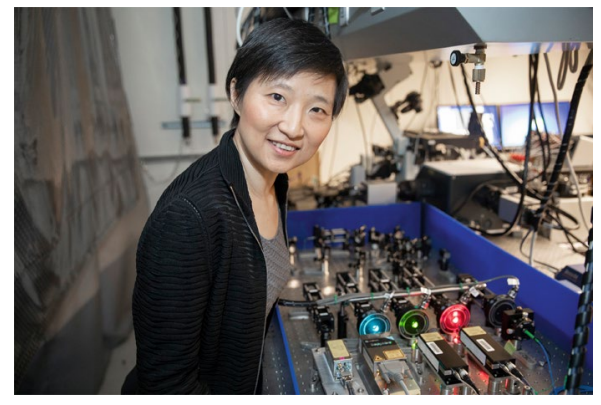

Xiaowei Zhuang developed the super-resolution microscopy method STORM. Single-cell genomics got her thinking about melding genomics and imaging. Credit: Harvard University

Drop-seq's barcoding scheme. They started out with 50-micrometer beads and moved to 10 -micrometer beads. Sam Rodriques in the Chen lab and Robert Stickels in the Macosko lab figured out how to arrange a bead monolayer as a 2D array and developed a protocol to transfer RNAs to the beads, which was "easier said than done," says Macosko. Slide-seq's output approaches that of single-cell analysis, which made it possible to use single-cell computational tools "as if the beads were single cells," he says. They have run single-cell analysis tools such as velocyto and Monocle. Among other projects, they have used the method to study development of the ocular lens and embryonic cortex. Lower-resolution spatial techniques have too many cells per pixel, which makes deconvolution more difficult, he says. Since Slide-seq uses beads, "we can continue to make the beads smaller and resolution better." The team has been working on the method's efficiency. Slide-seq v2 has mRNA capture efficiency that approaches that of scRNA-seq technology, he says. Efficiency matters, because for rare transcripts enough information is needed to confidently assign them to a particular location. Discussions on commercializing Slide-seq are in early stages, he says.

\section{Compute that space}

"The fact that it's not perfectly resolving every cell, I'm ok with that," says Elana Fertig, computational cancer biologist at Johns Hopkins University, although in an ideal world, she would want single-cell resolution. She works on tumor heterogeneity, which spatial techniques can help to resolve. One of her projects assesses $\mathrm{T}$ cell infiltration into tumors. The lab has a multi-omics focus, integrating data from different modalities, and the researchers use data from more than one spatial analysis method. She finds 10x Genomics' Visium technology more accessible than, say,
FISH. Molecular changes can define tumor types, but tumors can vary greatly within those classes. "Cell types aren't so binary," she says. She wonders if flow cytometry has shaped cell typing to date. Looking at samples with many markers for cells now might yield a continuum of cell identities. For this work, team science grows more important to iterate on findings and develop data analysis and visualization approaches to ferret out computational artifacts. The Fertig lab is part the National Cancer Institute's Informatics Technology for Cancer Research program to build the frameworks for interpreting the high-dimensional 'omics data that will increasingly include spatial data. With more markers per cell, the high dimensionality of the data have increased the challenges of data interpretation. "I think that's kind of the current challenge in the field for spatial," she says. For integrating imaging and single-cell data for spatial transcriptional analysis, she and her team apply transfer learning to overlay gene correlation signatures and perform dimension reduction.

Macosko foresees a phase of computational development around finding spatially variable genes or for finding trajectories that leverage the spatial information. Despite much effort, it remains a big challenge, says Shendure, to manage data and integrate across platforms. Each experiment leads to vast amounts of data, and researchers face decisions about what to save and what to discard and do not want to deviate from the principles of open data sharing in genomics.

\section{Data juggling}

At the Allen Institute, large-scale projects such as cell typing in the brain are as commonplace as juggling large datasets. It has even surprised them, says the Allen's Tasic, how quickly they can hit the boundaries of standard analysis when looking at single-cell and spatial transcriptomic data with tens of thousands of properties per cell. "The data matrices become unmanageable," she says. They reach out to work with others from outside biology who handle large-scale data matrices. The computational road ahead is daunting and exciting, she says. Her dream in graduate school of measuring all genes in all single cells is starting to come true. Beyond data size and dimensionality, says Zeng, she and her team work on integrating and correcting for batch effects. Even data captured with the same modality can differ slightly from one batch to the next and have to be normalized. To integrate across modalities, such as imaging, sequencing and electrophysiology, she and her team build 
clustering approaches and draw on different types of machine-learning methods.

Much opportunity exists, says van Oudenaarden, to computationally combine single-cell RNA-seq and spatial information to map single cells back onto space and to build and use reference maps and tools. $\mathrm{He}$ is intrigued by computational approaches to reconstructing spatial maps from say, in situ hybridization reference databases or approaches that use no maps. One such approach, novoSpaRc, does 'gene expression cartography' without maps. It's based on assumptions about how gene expression varies across a tissue section. Having ever more data about tissues is a nice problem to face, says van Oudenaarden. This wealth makes it "an even bigger puzzle" to get to the important "bones," the core aspects of biology. To him, the merging of single-cell 'omics and spatial methods is "really a way to generate hypotheses," he says. One needs to confirm and validate findings with data collected separately.

\section{Commercial takes}

Spatial technologies have a rich history, says Shendure, such as in the labs of Mats Nilsson, George Church and others, who have been working on projects such as genotyping or sequencing directly on tissue sections for well over a decade. Spatial analysis blossomed into a field in the past year or two, he says. That's in part due to the way the single-cell field has matured and technologies have been turned into commercially viable instruments with broad usage. "This last point is still one of the challenges, though," says Shendure. "Many of the most exciting methods are still somewhat bespoke and really only operational in one or a few labs," he says of spatial methods to date. The question, he says, is how more of these methods can be more readily adopted by other groups. When

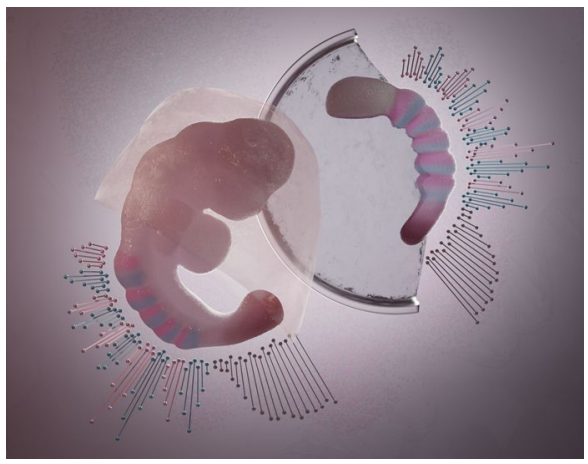

Spatially resolved transcriptomics reveals markers of somitogenesis expressed in stem-cell-based models of development as in mouse. Credit: A. van Oudenaarden, Hubrecht Inst. it's being done for the first time, much of what scientists do is difficult and expensive, says Zeng, but technology development helps to change that. Commercialization has been crucial in propelling single-cell sequencing, says Tasic, and the same can happen in spatial technologies. "I'm very much looking forward to a commercial solution for spatial transcriptomics that will work at the single-cell level," she says.

Spatial techniques, especially in situ techniques, "first took off probably the most in neuroscience," says Michael Schnall-Levin, 10x Genomics senior vice president of research and development. Other fields eager for spatial analyses are developmental biology, where people study gradients and spatial patterns of molecules in cells, and cancer research, especially for the assessment of tumor heterogeneity and $\mathrm{T}$ cell infiltration. Since it was founded, says Schnall-Levin, who has been with the company since its start, the teams began with microfluidic technology, long-read sequencing approaches and a focus on single-cell and spatial analysis. What began as a potential comarketing agreement with Spatial Transcriptomics turned into an acquisition in 2018. Visium is not yet single-cell resolution, but the company is working on ways to get there and "we're pretty far along," he says. He and his team also focus on in situ-based high-resolution analysis, says Schnall-Levin. The company acquired ReadCoor, a spinout of the Church lab at Harvard Medical School, and Cartana, a spinout from the lab of Stockholm University and Science for Life Laboratory researcher Mats Nilsson. Cartana uses padlock probes that hybridize to the mRNA of genes of interest, followed by rolling circle amplification to amplify the RNA on-site to prepare it for sequencing. ReadCoor uses barcoding and probes for fluorescence in situ RNA sequencing and protein detection, too. Cartana brings to 10x Genomics an intellectual property portfolio, chemistry and scientists with know-how, which is a seed one can build around in an accelerated fashion, says Schnall-Levin. ReadCoor brought scientists, a "foundational technology," intellectual property and technology development, since the team that built the company's instrument had worked though some "non-trivial" engineering constraints, he says. Between the two companies $10 \mathrm{x}$ received 110 patents, leading to a company total of 935 patents, says Shernaz Daver, who advises 10x Genomics. These technologies enable pinpointing molecules down to subcellular locations, says Schnall-Levin. For now, they measure sets of a few hundred genes, but "obviously, that has room to grow." He views

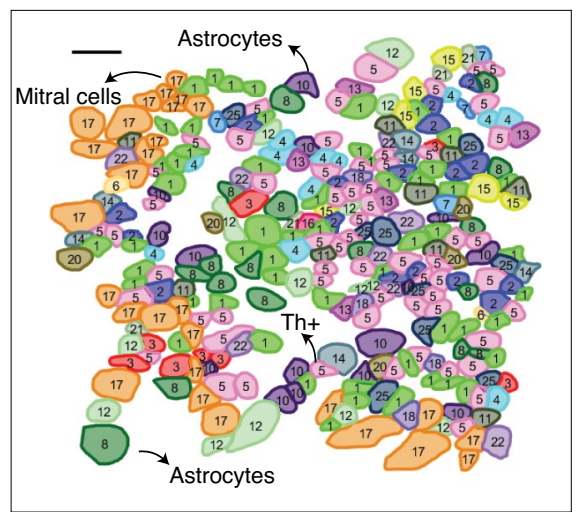

Spatial techniques can reveal a tissue's complex cell type mixtures - here, the mouse brain's olfactory bulb. Credit: Cai lab, Caltech, I. Strazhnik Adapted with permission from ref. ${ }^{6}$, Springer Nature.

the approaches from ReadCoor and Cartana as complementary to the technology underpinning Visium. The company keeps relations with academic labs after acquisitions, since they have expertise that is important for guidance and it's part of being "good citizens" of the research community.

In 2019, nanoString began selling its instrument, GeoMx, for spatially resolved tissue analysis. The device had been in testing with early adopters. Some labs worked with prototypes and others sent their samples to the company's Seattle headquarters and used the instrument remotely. "We learned so much from that," says Joe Beechem, chief science officer and senior vice president of research and development. "They're sitting in their living room with a glass of wine, controlling it, laughing like little kids." Early on, GeoMx could capture around 100 mRNAs; now that number is over 22,000, which shows how fast this field is moving, says Brad Gray, nanoString president and chief executive officer. nanoString launched as a spinout from the Institute for Systems Biology in Seattle with technology for optical molecular barcodes and is now, says Gray, all about spatial analysis. Early adopters of the GeoMx platform were mainly in immuno-oncology, where labs sought to see, for example, whether $\mathrm{T}$ cells were trapped in the periphery of a tumor instead of bringing their destructive power deep inside it, says Beechem. One day, right before giving a talk, he scribbled on a napkin how the company's oligo-barcodes could spatially resolve tissue and deliver a readout on a second-generation sequencer. The company had previously developed a way to attach barcodes to antibodies with 
photocleavable linkers. His idea was to spread the linkers and barcodes on a tissue slide. The oligos hybridize to mRNAs. A microscope takes snapshots of the tissue and then emits a burst of light, which pops off the photocleavable linker. The mRNAs and barcodes are then sequenced. As Gray explains, their customers have long requested ways to study whole transcriptomes and gain higher resolution down to compartments in cells so they can ask: "Is it on the surface, is it in the nucleus, is it attached to certain organelles?" The market, in his view, is going to split into two instrument classes. There will be profilers such as GeoMx and Visium that look at multicellular regions of interest in high throughput. Then there will be instruments with cellular and subcellular resolution, which may or may not cover the whole transcriptome but will cover thousands of genes at a time. nanoString has just launched its GeoMx Digital Spatial Profiler, which has single-cell resolution. Gray knows nanoString's competitors include Fluidigm's Hyperion Imaging System, an imaging mass cytometer, and Ionpath's MIBIscope. The new nanoString technology also uses photocleavable tags for gene-expression profiling of tissue, including formalin-fixed paraffin-embedded tissue. It is being applied, for example, to study spatial aspects in autopsy samples from people who died of COVID-19. With technology of this type, says Beechem, researchers will have all the power of all the molecular tools they had in the past; "they can just do it in space now."

At BGI Research, a high-resolution method for spatially resolved gene expression analysis is under development. The idea is to bridge the gap between single-cell sequencing and spatial analysis of where cells sit in tissue, which together will shape the study of function as well as structure, says Xun Xu, CEO of BGI Group, who directs BGI Research. He watches other company activities in this area and believes the BGI approach will have the highest resolution. The approach captures a few hundred data points per cell, says Xu. It's packaged on a silicon chip as a nanoarray built with photolithographic techniques adapted from the semiconductor industry. BGI has used this approach to profile DNA-protein interactions, and Chris Ao Chen, a scientist at BGI Research, says he and his colleagues are now working on ways to embed cells or tissues on these chips to yield high-resolution spatially resolved data. The square compartments of the silicon chip's array each hold round areas, each containing barcoded DNA nanoballs resolvable with conventional wide-field imaging. DNA nanoballs are bundles of

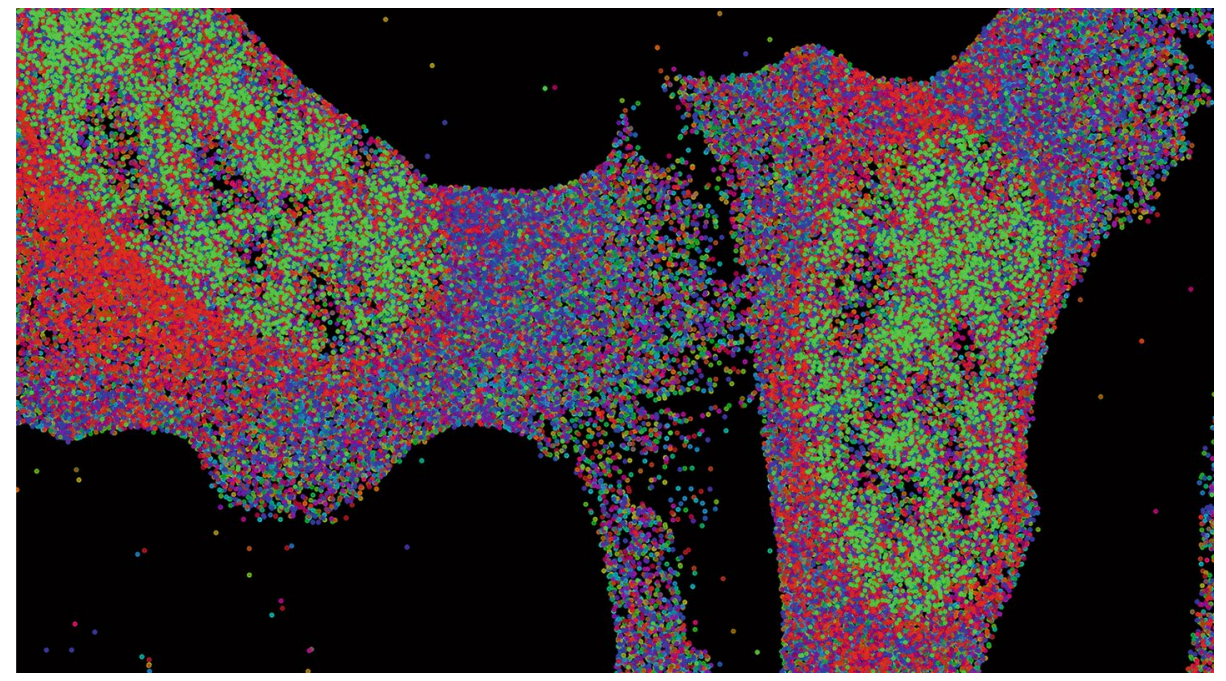

With MERFISH, the Zhuang lab captured the expression of 10,050 genes in individual cells in human cancer cells. RNA molecules from different genes are shown in different colors. Credit: X. Zhuang laboratory, Harvard U./HHMI

circularized cDNA made from captured transcripts and include primers and adapters ready for BGI's array-based sequencing. Each bar-coded readout has as its spatial address a specific pitch on the array. The tracking lines on the chips give the coordinates of what is measured in each cell, says $\mathrm{Xu}$. The spatial method draws on BGI's acquisition of technology from Complete Genomics, Inc. and is slated for release sometime in 2021. BGI has made chips for spatial analysis of mouse brain with around 2,000 genes and 4,000 gene transcripts per nanoball bin. The team is thinking beyond mRNA to other 'omics, says Xu. Ultimately the instrument can help researchers build human and model organism reference tissue maps; they might use this spatial technique to assess how genetic variants affect tissue function, such as in cancer, or use it for cross-species comparative approaches, such as tissue-based studies of evolution and adaptation of organ structure.

Companies involved in spatial areas are active software developers. Informatics has been a core aspect of the company, says Schnall-Levin, whose team is committed to open-source software. Researchers can add their algorithms and tools to the $10 \mathrm{x}$ Genomics pipeline. As Xu explains, the team has worked on interactive visualization tools "to give people a real view of the tissues." nanoString's Gray says, "Joe's largest department within R\&D is now software." The company has more programmers than molecular biologists and engineers.

\section{Atlas at hand}

Spatial analysis is crucial for the Brain Initiative Cell Census Network (BICCN), a project funded by the US National Institutes of Health in which many labs work together to produce reference human, mouse and non-human primate brain atlases. It involves imaging, electrophysiology, and molecular genetic analyses such as transcriptomics and epigenomics. Work on the Allen Mouse Brain Atlas began with situ expression profiling of all mouse genes to show anatomical and spatial gene expression patterns. "It's a reference database that has been widely used," says Zeng. She directs BICCN's whole-brain atlas ventures for the mouse brain. The team is building a "periodic system" of cell types in the mouse and human brain, says Tasic. She leads a BICCN project on cell-type-specific tools for brain-wide labeling and ways to study circuits in cell-type-specific ways. The research community will soon have cell-type-specific reference atlases onto which they can map their own findings. Among other papers, the BICCN teams recently published a multimodal cell census and atlas of the mammalian primary motor cortex $^{8}$. BICCN phase two is starting, says Zeng. Among the new projects are ones devoted to cell-type targeting tools to apply spatial and molecular information, perturb cells and characterize their roles in a circuit and behavior. "That's how you build causality," says Zeng.

For the recent atlas of the mouse primary motor cortex, the teams note that single cell genomics has swept across biology, including neuroscience, and catalyzed a transformation from labs being able to classify and describe phenotypes to having a mechanistic and explanatory molecular genetic framework for the cellular basis of 
brain organization, which includes complete transcriptomes. For mapping the spatial organization of more than 300,000 cells from the mouse's primary motor cortex by transcriptomic cell type, they chose Zhuang's MERFISH ${ }^{9}$, multiplex error-robust fluorescence in situ hybridization. They combined MERFISH with other methods such as Patch-seq, in which cells are measured with electrophysiology and then sequenced. They analyzed and compared mouse, marmoset and human transcriptomic cell types. Then they selected a panel of over 250 genes to image and then cluster by single-cell expression profiles, finding "excellent correspondence" with clusters identified by single-nucleus and single-cell RNA-seq. This let them discern the spatial distribution of cell types and gain a view of cortical layers that refined traditionally defined layers. They also noted spatial distributions along medial-lateral and anterior-posterior axes. On occasion, disagreements erupt about the brain's cartography, but Tasic believes advancing technologies will slowly help to settle these disagreements, which are sometimes based on labs using different methods. With a common coordinate system in place, the disagreements can shift, for example, to the necessary discussion about function. "Very often one gene is not sufficient to identify a cell type," says Zeng. "Black and white frequently does not exist," says Tasic in terms of cell types. "You have multiple genes that are expressed at multiple different levels and together they make a cell what it is." The ability to look at thousands of genes expressed in individual cells "has already changed the field dramatically, says Zeng. Spatial transcriptomics adds richness: "maybe not thousands of genes yet," depending on the method, but it's the same idea: researchers get a view of many of a cell's genes in a spatially localized way.

Zhuang is happy MERFISH is being used for brain atlas building. "I'm just personally very fascinated by the brain," she says. In MERFISH, RNAs are barcoded during hybridization rounds. Non-fluorescent targeting probes bind the mRNA and fluorescent readout probes hybridize to the targeting probes. The readout sequences yield a barcode distinct to each mRNA. The sequential rounds of hybridization, the barcoding and error correction make it possible to capture many genes. The challenge with hybridization rounds, however, is the accumulation of errors, says Zhuang. Each round has a small error rate individually, but the errors accumulate and become significant, which risks gene misidentification. smFISH has high accuracy, says Zhuang. But it's a one-time

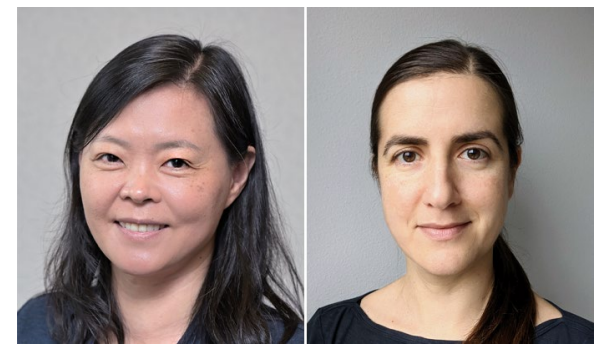

Hongkui Zeng (left), director of the Allen Institute for Brain Science, and Bosiljka Tasic, also at the Allen Institute. Credit: Allen Institute for Brain Science

measurement, not a sequential measurement that accumulates errors. As she and her team developed MERFISH, they worked on imaging, built-in error correction and a way to deliver results on thousands of RNA species transcriptome-wide. "That took us a while," she says. The method achieves $80 \%$ detection efficiency. In a separate BICCN-based project, Zhuang and her team used MERFISH to analyze more than one million cells in the preoptic region of the mouse hypothalamus. In the brains of female mice that had not previously given birth to pups, exposure to pups activated a neuron that is also activated in female and male mouse parents but not active in males that have not fathered a litter. She and her team also apply MERFISH to characterize subcellular spatial organization to reveal RNAs in different cellular compartments and the way chromatin is organized ${ }^{10}$. Ultimately this work shows "how 3D genome organization and transcription are related to each other," she says. Along with Harvard Medical School researcher David Walt and Jeffrey Moffitt, a former postdoctoral fellow of hers who now has his own Harvard Medical School lab, she has founded a company, Vizgen, to commercialize MERFISH.

\section{Future in space}

As a microscopist, Zhuang is happy to see the fields of imaging and single-cell genomic analysis grow closer. KTH's Ståhl looks forward to high-resolution spatially resolved transcriptomics. "Maybe, the single cell, like ten micrometers, may be the sweet spot," he says, that delivers enough data per pixel. Zeng is happy about the useful spatial analysis techniques that have emerged. Many of the existing methods lead up to single-cell RNA sequencing, which still gives the highest sensitivity readout for quantifying gene expression. One day, though, she hopes "we can just get completely rid of single-cell sequencing" for transcriptome-wide single-cell spatial analysis.
Spatial information clearly matters, says Shendure, "and we're still in the early days as far as where one can imagine the technologies eventually going." It's exciting that spatially resolved transcriptomics is the Method of the Year, says Macosko, and "I think it's really just the beginning." To dig into a question of interest, people will likely use several of the many tools and techniques in the spatial analysis field. Standards, metrics and benchmarks will help the spatial field, just as they are helping the single-cell genomics field, he says.

What started with single molecule FISH and single-cell genomic analysis has become a group of ways to bring the large-scale hypothesis-generating technologies of genomics into cell biology and tissue biology - one that will ground thinking and technology development, says Macosko. Researchers will be able to do fine-grained studies of gene expression in a spatial context and make other measurements, too, such as of enzymatic processes and interactions between cells, among genes and between proteins. They will be able to barcode and anchor aspects of interest in space, and this stands to change the way cell biology as well as pathology and histology are practiced, he says.

Zeng sees many future opportunities. "The techniques are not, in many cases, they are not perfect yet," she says. "We want more and better," she says, to scale up techniques and be able to measure thousands of genes routinely and efficiently. Spatial methods will become standard, says Tasic. One day people will send out tissue and get back spatially resolved single-cell genetic information, she says. Commonplace reactions to papers in this area might become: "Oh, you didn't measure all the genes? Why not?"

Vivien Marx ${ }^{凶}$
Nature Methods.
${ }_{\text {e-mail:v.marx@us.nature.com }}$

Published online: 6 January 2021

https://doi.org/10.1038/s41592-020-01033-y

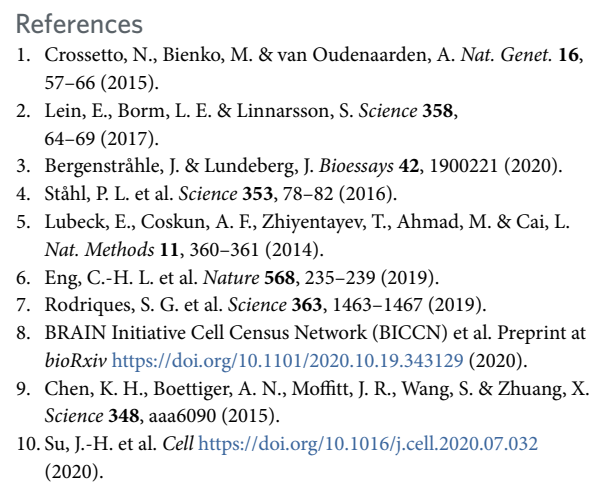

References

Crossetto, N., Bienko, M. \& van Oudenaarden, A. Nat. Genet. 16 57-66 (2015)

\& Linnarsson, S. Science 358

4. Ståhl, P. L. et al. Science 353, 78-82 (2016).

5. Lubeck, E., Coskun, A. F., Zhiyentayev, T., Ahmad, M. \& Cai, L. Nat. Methods 11, 360-361 (2014)

6. Eng, C.-H. L. et al. Nature 568, 235-239 (2019).

7. Rodriques, S. G. et al. Science 363, 1463-1467 (2019).

BRAIN Initiative Cell Census Network (BICCN) et al. Preprint at bioRxiv https://doi.org/10.1101/2020.10.19.343129 (2020).

Science 348, aaa6090 (2015).
10. Su, J.-H. et al. Cell https://doi.org/10.1016/j.cell.2020.07.032 (2020). 